\title{
NEW APPROACH TO TRAFFIC DENSITY ESTIMATION BASED ON INDOOR AND OUTDOOR SCENES FROM CCTV
}

\author{
Agata CHMIELEWSKA*, Marianna PARZYCH*, \\ Tomasz MARCINIAK*, Adam DĄBROWSKI*
}

\begin{abstract}
In this paper we present an algorithm for precise estimation of moving objects density (typically people and vehicles) in indoor and outdoor scenes. Automatic generation of the so-called density maps is based on video sequences acquired by surveillance systems. Our approach offers two types of solutions. The first one increments the accumulation table when a moving object is detected in a location of interest, delivering a density map of the presence of moving objects. The second algorithm increments the accumulation table only in cases of detecting a new moving object, resulting in a density map of the count of moving objects. The proposed algorithms were tested with the use of PETS 2009 database and with our own database of long-term video recordings. Finally, results of the density maps visualization and determination of the "busy hours" are presented.
\end{abstract}

Keywords: moving objects density estimation, traffic estimation, density maps, behaviour analysis, marketing spaces analysis, indoor and outdoor video surveillance

\section{Introduction}

Analysis of people behaviour and determination of the size of the crowd in public spaces is becoming increasingly important in the video monitoring systems. In recent years we have seen many accidents at social events. Panic and improperly designed spaces, occurrence of the so-called "bottlenecks" in evacuation ways, are common causes of an increase in the number of casualties.

Another area that uses analysis of people behaviour is the field of marketing. Despite the development of online shopping, physical distribution of products remains the most important channel of the customer service. Psychological analysis of the consumer behaviour in sale rooms aims at increasing the sale success. Based on even short-time observations, consulting companies can draw conclusions that can effectively improve functioning of the sale area.

* Faculty of Computing, Poznan University of Technology, Poznan, Poland Corresponding author e-mail: marianna.parzych@put.poznan.pl 
Conventional solutions for the people behavior analysis in the sale rooms require the use of manual analytical tools like questionnaires or physical traffic counting by interviewers. Such methods are cumbersome and expensive, thus impossible to operate in a large number of rooms over a long time. An interesting and attractive option is the use of the CCTV (closed circuit television) systems, since most commercial spaces are equipped with a video monitoring. Thanks to this, manual observation can be supported by advanced automatic analysis of video sequences.

A subject of this paper is the automatic generation of density maps of moving objects (people and vehicles). The proposed solutions allow to draw conclusions about changes in the moving objects density distribution at the studied regions of interest (increasing and decreasing the moving objects density in particular areas, formation and movement of queues, occupation of service desks, availability of exits and evacuation routes, traffic optimization in various indoor and outdoor situations) all without long hours of manual video analysis.

Two particular solutions are proposed. The first one consists in incrementing the so called accumulation table when a moving object is detected in a location of interest, delivering a presence of moving objects density map. It may operate in real time showing the results with specified time intervals, for example every hour. These results can e.g. be used by companies to draw conclusions needed to increase sales based on short-term observations of clients.

The second solution, not yet available in contemporary commercial CCTV systems, consists in incrementing the accumulation table only in case of detecting a new moving object, resulting in a count of moving objects density map type.

\section{Related work}

The density maps generation is directly related to the moving objects analysis. Typical solutions are based on the analysis of stationary scenes from the video sequences. Estimation of crowd density is provided in [11] with the use of the texture information based on grey level transition probabilities. People detection in [10] is based on the Haar wavelet transform (used for the head detection) and on the support vector machine classifier. The authors of [20] proposed the translation invariant orthonormal Chebyshev moments method for pattern recognition.

Other approaches assume collecting data about the movements of objects. In [18] and [6] the head detection based on the HOG (histogram of oriented gradients) transform is extended with the optical flow approach. Density maps can be built by accumulation of BLOBs (binary large objects) of moving people. The authors of [9] use the optical flow for foreground objects detection. Generation of motion maps applied to the crowd monitoring system on the escalator is presented in paper [22].

The estimation of density can be used as a part of larger surveillance systems. Rodriguez et al. [21] use information about the density to support the tracking algorithms in crowded scenes. The solution described in [24], based on 3D images from the time-of-flight camera, allows controlling the density of the crowd in real time to assess the safety in the public spaces. The camera that is used reduces the range of vision in the system, which is ideal for small spaces, tunnels, narrow passages, etc. 
Our method for estimation of people density is based on an alternative approach. We use the information about trajectories of moving objects and generate maps that show the people density per time unit, averaging the instantaneous density in the individual frames.

It should be mentioned that generation of density maps based on data from video monitoring is already offered by several companies, e.g. by: Mobotix [14], Prism Skylabs [23], Bosch [1], and Cognimatics [7] but all of them are subject to important restrictions.

The software from Prism Skylabs visualizes customers behaviour, the density maps are generated, and the results are also visualized in a form of time line, which shows motion activity levels in the specified periods of time. A disadvantage of this method is that the results are not scalable to more general data (e.g. for a whole hour or for a whole day). This disadvantage is resolved in the present paper by means of the proper data normalization.

Another available tool is MxAnalytics byMobotix company. This software collects statistical data of moving objects, for example counts people and generates density maps with the use of tracking moving objects contour lines. The camera records how often each contour line is passed over within a specified period. The most frequented areas are highlighted in colour in the density map. This software uses, however, a linear reference, thus the colors are sometimes wrongly mapped.

The IVA 5.6 software from Bosch does not generate the density maps, which is a drawback, but it tracks the moving objects and stores paths of movements of these objects. It also deals with the crowd density estimation (by counting the objects).

\section{Test video sequences}

In order to assess performance of the proposed methods for density estimation, experimental studies were performed. During tests three sets of recordings were used: PETS 2009 database and two authors' databases: video sequences recorded in the sales rooms and those recorded in the parking lot in front of a large shopping centre.

\subsection{PETS 2009 database}

PETS 2009 (performance evaluation of tracking and surveillance) database [19] has been developed for advanced image processing (e.g. for analysis and estimation of the human silhouette, human movement, and number of people in the crowd). Datasets in the PETS 2009 database come from multiple cameras placed in different positions, representing different behaviours of the crowd (the number of people is about 40). This database consists of the following modules: training data, images used for person counting and people density estimation, people tracking, flow analysis of the crowd, and event recognition. Each dataset contains several sequences and each sequence contains different views. Examples of scenes used in this paper for people detection and tracking are shown in Fig. 1. The cameras used for recording the datasets are: Axis 223M, PTZ Axis 233D, Sony DCR-PC1000E 3xCMOS and Canon MV 1 1xCCD. A resolution of the video 
frame is $768 \times 576$ pixels with the frame rate of $7 \mathrm{fps}$ (frames per second). Frames are compressed as JPEG image sequences (the motion JPEG format).

As already mentioned our approach to people density estimation assumes determination of density per time unit. In order to obtain density maps, recordings of different lengths are required. The PETS 2009 database and other databases of surveillance recordings contain mostly short sequences (about few minutes). For this reason, they do not fully meet the requirements necessary for our approach.
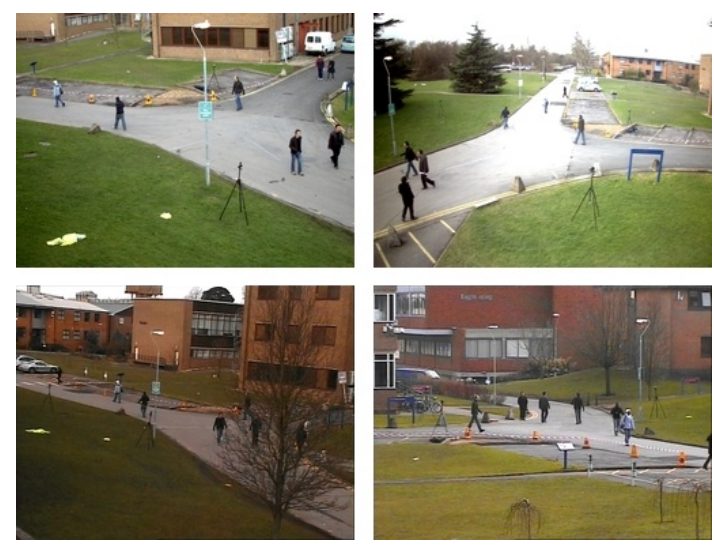

Figure 1. Sample images from the PETS 2009 database

\subsection{Sales room test sequences database}

Because of the problems described above we have prepared our own database and test sequences. Recordings were carried out under real-life conditions in commercial customer service points and sales rooms in four locations. During recordings the KTC3510 color camera from KT-VISION with TAV308DC 3-8 mm SIRIUS F lens were used. The camera was located in the corner of the room near the ceiling. This camera arrangement allows for a good observation of people positions and has a quite positive impact on quality of the moving objects detection algorithm. The database consists of 64 hours of recordings made within 8 days (with 25 fps, with $704 \times 288$ pixels resolution), coded with H.264 codec. The sample video frames are shown in Fig. 2.

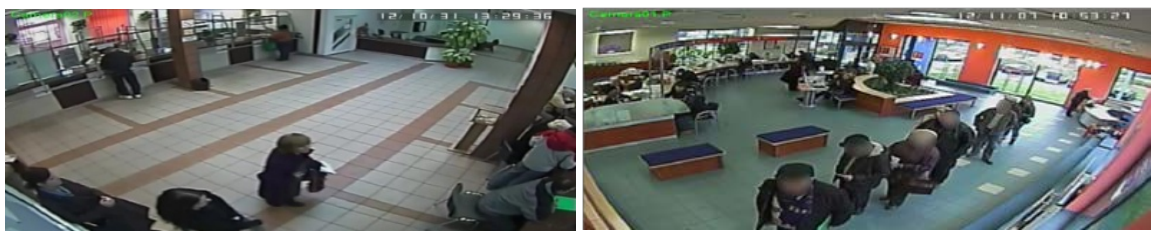

Figure 2. Sample images from the authors' sales rooms database 


\subsection{Parking lot test sequences database}

The database of outdoor video surveillance situations was also prepared. During recordings NV-GS500 color camera by Panasonic was used [15]. Figure 3 shows illustrative images from video sequences used during tests in the outdoor scenes. Images show the observed parking lot. Recordings were carried out in front ofthe Green Point shopping center in Poznan under real-life conditions. The camera view includes a parking lot, an entrance to the shopping center and other retail outlets. The location of the camera was high above the parking lot. By this means the moving objects were entirely covered, which affects the proper objects detection and tracking. The Green Point shopping center in the day of recordings was open from 6 AM to 9 PM. For this reason 8 one hour length video recordings were registered, starting from 6 AM with hourly intervals.
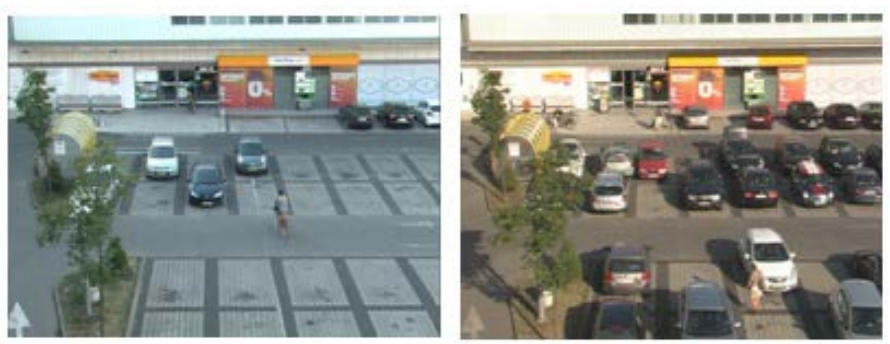

Figure 3. Sample video frames from the authors' parking lot database

\section{Density map estimation}

\subsection{Moving objects detection and tracking}

Our algorithm is based on the "Motion-Based Multiple Object Tracking" model from the Matlab [2] environment. This model is constructed with the use of Gaussian mixture models (for background subtraction), morphological operations, and BLOB analysis (performed to connect pixels corresponding to adequate moving objects). Motion of each person is estimated and predicted with the Kalman filter [5] in order to track them continuously. The vision.KalmanFilter [3] module is used. The Kalman filter object is configured and created with the use of the configureKalmanFilter function [4]. The vision.KalmanFilterMotionModel parameter is set to 'ConstantVelocity', the InitialLocation parameter is set to the location where the moving object was detected for the first time. The InitialEstimateError and MotionNoise parameters were customized. Details about the parameters adjusted for improvement of efficiency of people tracking are listed in Table 1. 


\subsection{Proposed method for density analysis}

The proposed method for people density and traffic analysis is an extension of the algorithm described in our previous paper [16]. As it was already mentioned in Introduction it is based on generation of two types of density maps. The first type is called the time (or presence) density map (TDM). The second type is called the count density map (CDM). Both types describe how long moving people or vehicles have occupied prescribed areas of the scene but with the latter approach the objects are tracked and the relevant individual objects are taken into account only.

Table 1. Parameters of the "Motion-based multiple objects tracking" algorithm

\begin{tabular}{|c|c|c|c|}
\hline Algorithm module & Algorithm's parameter & Default & Adjusted \\
\hline \multirow{2}{*}{$\begin{array}{l}\text { object: } \\
\text { vision. } \\
\text { ForegroundDetector }\end{array}$} & NumTrainingFrames & 40 & 20 \\
\hline & $\begin{array}{c}\text { Minimum } \\
\text { BackgroundRatio }\end{array}$ & 0.7 & 0.65 \\
\hline \multirow{3}{*}{$\begin{array}{l}\text { object: } \\
\text { vision. } \\
\text { BlobAnalysis }\end{array}$} & MinimumBlobArea & 400 & 800 \\
\hline & MaximumBlobArea & - & 10000 \\
\hline & Connectivity & - & 4 \\
\hline \multirow{2}{*}{$\begin{array}{l}\text { function: } \\
\text { detectObject }\end{array}$} & Imclose('rectangle', x) & $\mathrm{x}=[15,15]$ & $\mathrm{x}=[3,3]$ \\
\hline & Imerode('rectangle', y) & - & $\mathrm{y}=[3,3]$ \\
\hline \multirow{2}{*}{$\begin{array}{l}\text { function: } \\
\text { deleteLostTracks }\end{array}$} & invisibleForTooLong & 10 & 12 \\
\hline & ageThreshold & 8 & 5 \\
\hline \multirow{3}{*}{$\begin{array}{l}\text { function: } \\
\text { createNewTracks }\end{array}$} & configure & {$[200,50]$} & {$[100,50]$} \\
\hline & KalmanFilter & {$[100,25]$} & {$[50,25]$} \\
\hline & ConstantVelocity & 100 & 25 \\
\hline
\end{tabular}

A process of calculating the density maps is as follows. Each detected moving object (the BLOB area) is described by coordinates of the rectangle vertices surrounding it. Then the center of the rectangle of each person in case of indoor scenes and the bottom part of the moving objects in case of outdoor scenes (including cars) is calculated. In the case of the object detection in the video sequence, the corresponding cell in the two-dimensional accumulation table is incremented under assumption that each blob corresponds to a single object a person or a vehicle).

After the processing of the entire video sequence the density map is generated based on the values in the accumulation table. The maximum value in the accumulation table is searched and according to this value, the whole accumulation table is scaled to values in the range $\langle 1,255\rangle$ [17]. After the accumulation table scaling, the values, with the number of objects passed through a given point or the time, in which a person was at that point, cannot be correctly read, but visualization of results in the image with the use of 2D histogram are better and more intuitive. The next stage is the reference image loading, which has resolution of $N \times 255$ pixels. Each value in the accumulation table (after scaling) corresponds to color in the adequate line in the reference image. Thanks to these operations it is possible to visualize object density maps using appropriate color scales.

There are many different color scales used in practice. To optimize a visualization effect, we have chosen two of them: the standard, so called rainbow color scale in Fig. 5 
and a nonstandard (our) color scale (starting from navy blue, through purple, pink, yellow, and finishing with white) used in Figs. 6 and 7. The rainbow color scale in Fig. 5 is linear but two versions our color scale are used: the nonlinear (logarithmic) version in the left hand side of Fig. 6 and the linear version in the right hand side of Fig. 6 and in Fig. 7. All these variants are chosen for equalization of the final visualization effects in these figures.

In the TDM case, cells in the accumulation table are incremented every time when a moving object is detected in a given cell. The CDM algorithm accumulates cells in the table only once for each moving object in a given location. This kind of the density map shows the paths that moving objects follow. The whole process of generating the density maps is presented in Fig. 4.

The results obtained with this algorithm allow for evaluation of the distribution of the density of moving objects, but do not provide numerical results such as the number of moving objects in a given area. However, an extension of this system with a module for example for counting people entering and leaving a room, as described in $[8,12,13]$, allows to obtain this information.

\section{Results}

The proposed algorithms were implemented in the MATLAB environment. During tests Intel(R) Core(TM) i7 CPU @ 2,7 GHz was used. The processing time of even nonoptimized (in terms of the time-consuming calculations) MATLAB code allows for real time operation. For a sample of 100 frames we obtained the processing speed of $47 \mathrm{fps}$.

\subsection{Case-study for indoor video surveillance application}

To verify the effectiveness of moving objects tracking and density maps generation, the algorithm was tested with the use of the recordings from different sale rooms. Density maps analysis from an hour-long videos allows to observe changes in the people distribution in the room during the day - movement of queues, density of people at service points. Figure 5 shows examples of the density maps (TDM and CDM) produced during a one working hour of one day and for one location.

On the basis of the TDM (Fig. 5a) it is possible to distinguish 1 PM as an hour, when a particularly high density of people occurred (rush hour). A distinct change in the areas of different levels of density can be observed. It may be noted that the size of the area with an intermediate density of people is significantly larger than the size of area with low density.

On the basis of the CDM (Fig. 5b), the highest density also occurs at 1 PM. For this rush hour the size of the area occupied by people is only slightly larger than beyond that hour, however a clear decline in areas with a low density and an increase in the area with the intermediate density can be seen.

Combining the conclusions based on both types of maps, it is possible to notice a movement of people in the direction towards the service point at the end of the room. It can also be noted that during the day most people in the room are at the entrance, which 
makes getting in and out of the room difficult. From the outside it gives an impression of crowd, which discourages next potential clients and can be very dangerous if a sudden evacuation would be needed, because of possible panic.

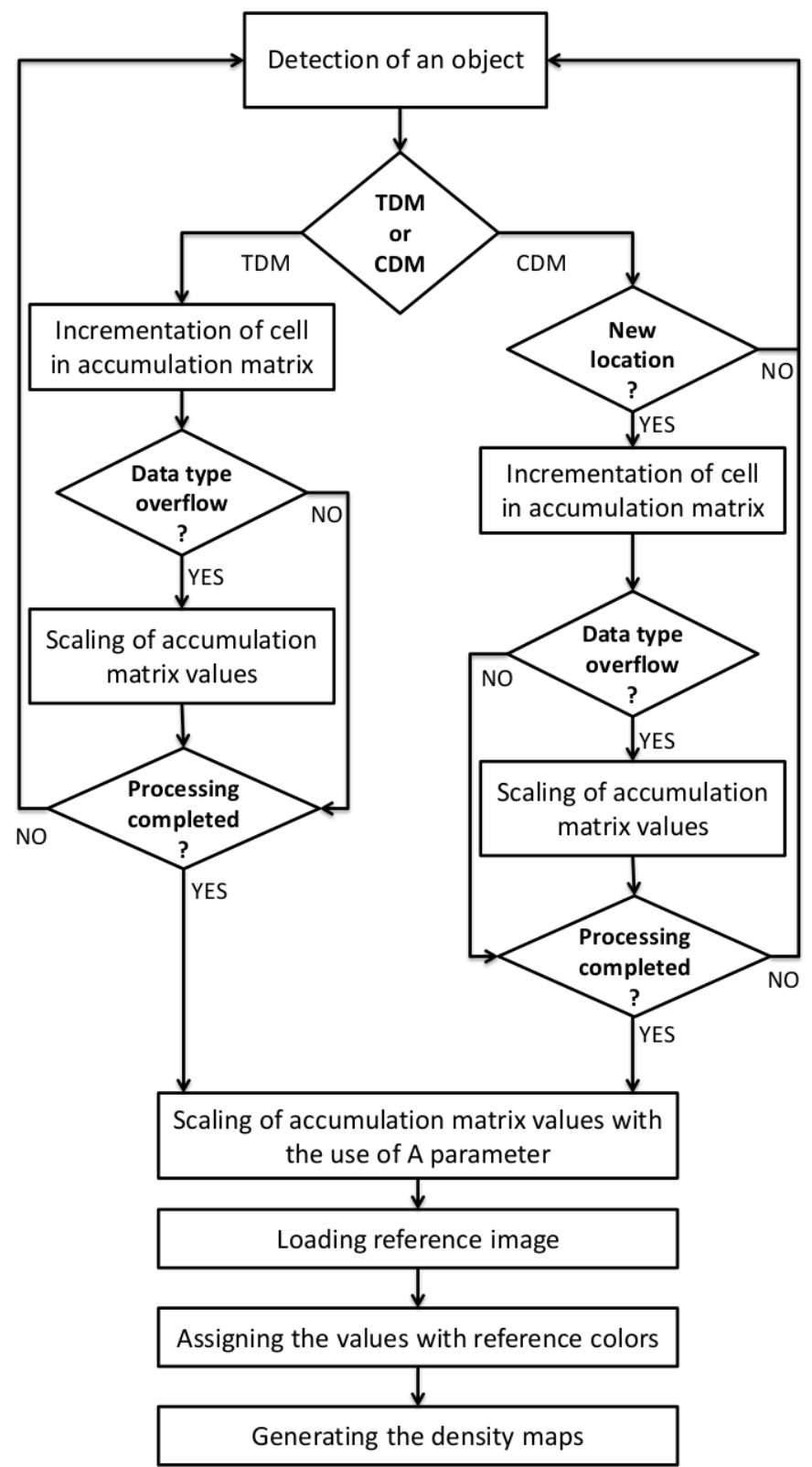

Figure 4. Particular steps of density maps generation 


\subsection{Case-study for outdoor video surveillance application}

Figure 6 shows examples of density maps generated in front of the Green Point shopping center parking lot. Moving objects movement distributions are clearly visible. The density of movement increases every hour (up to $6 \mathrm{PM}$ ). It can clearly be seen that the place near to the entrance to the shopping center is the most occupied area. This analysis allows to conclude that the entrance to this shopping center is the bottleneck. From the distribution of pedestrians passing between cars, we can conclude that the pedestrian crossing in the parking lot is located in a wrong place and should be moved to the right according to the camera point of view.

In order to improve the data visualization, the data from the accumulation table from one hour-long video sequences was normalized with the use of the whole day highest result. The normalized data can be interpreted by the system user in more intuitive way (Fig. 7). The system user can easily distinguish results with the lowest density of moving objects.
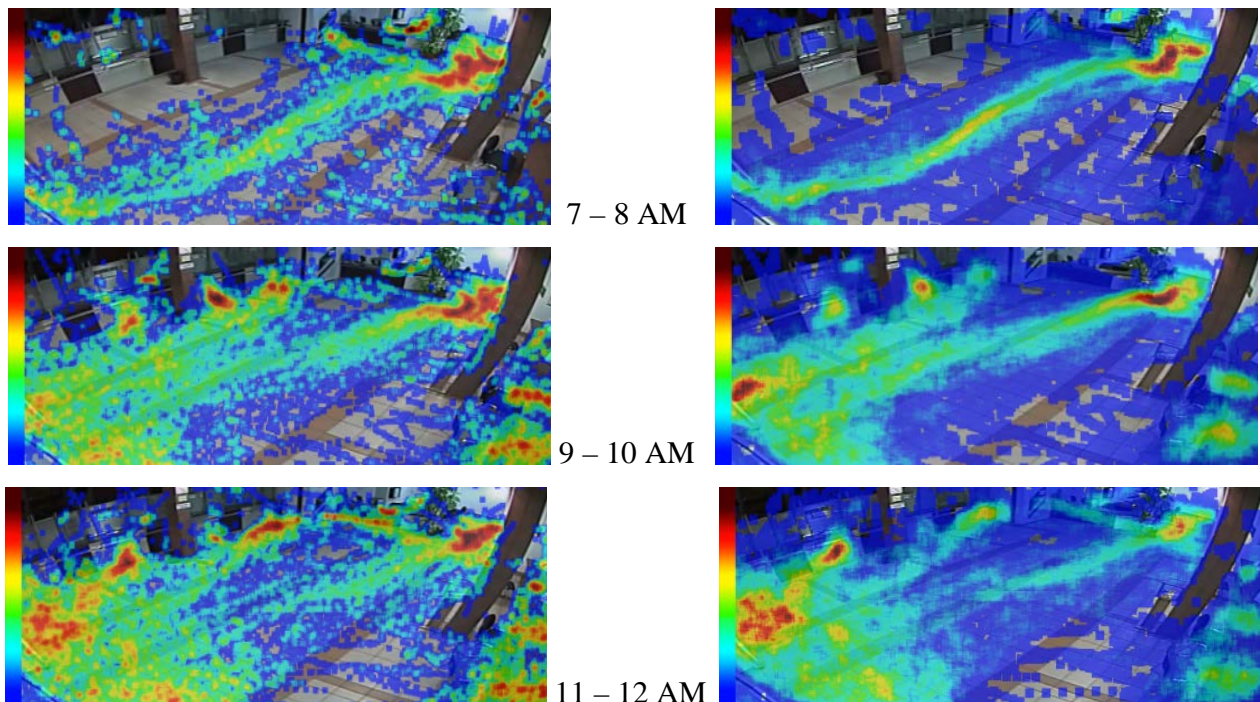

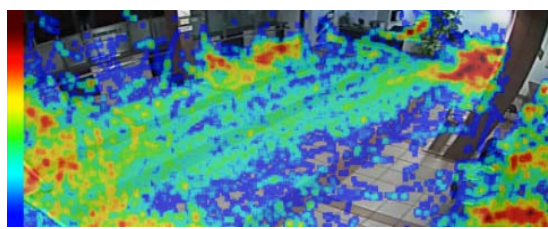

(a)

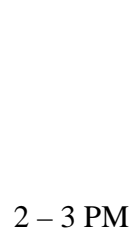

$2-3 \mathrm{PM}$

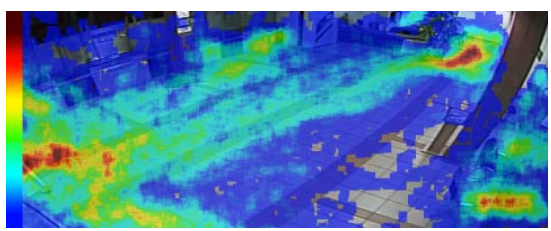

(b)

Figure 5. Selected examples of people density maps: (a) TDM type and (b) CDM type between: 7:00 AM and 3:00 PM 

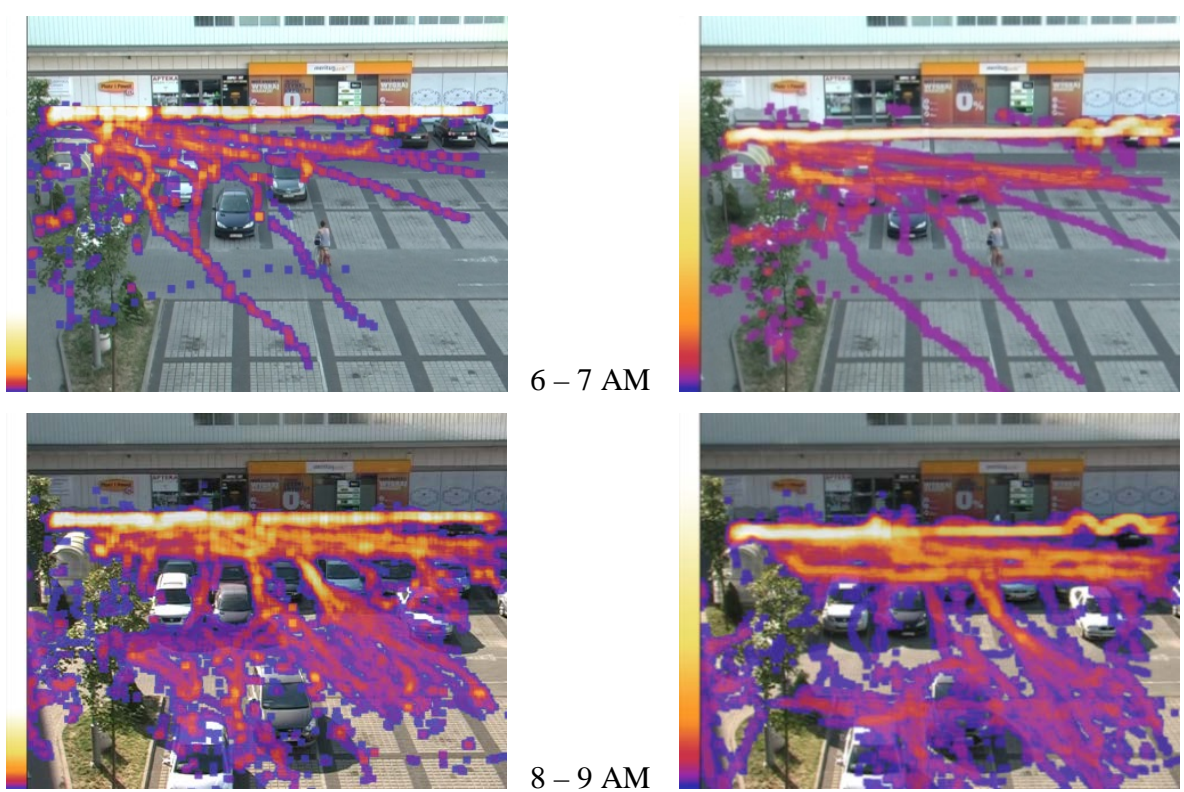

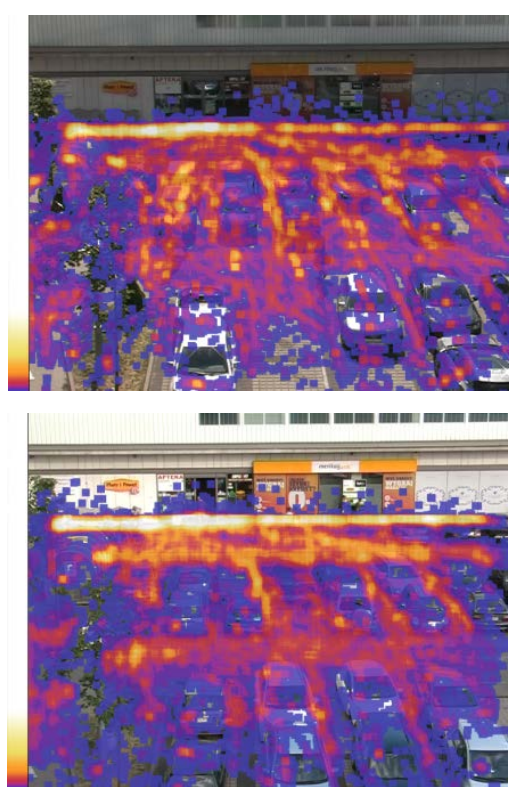

(a)
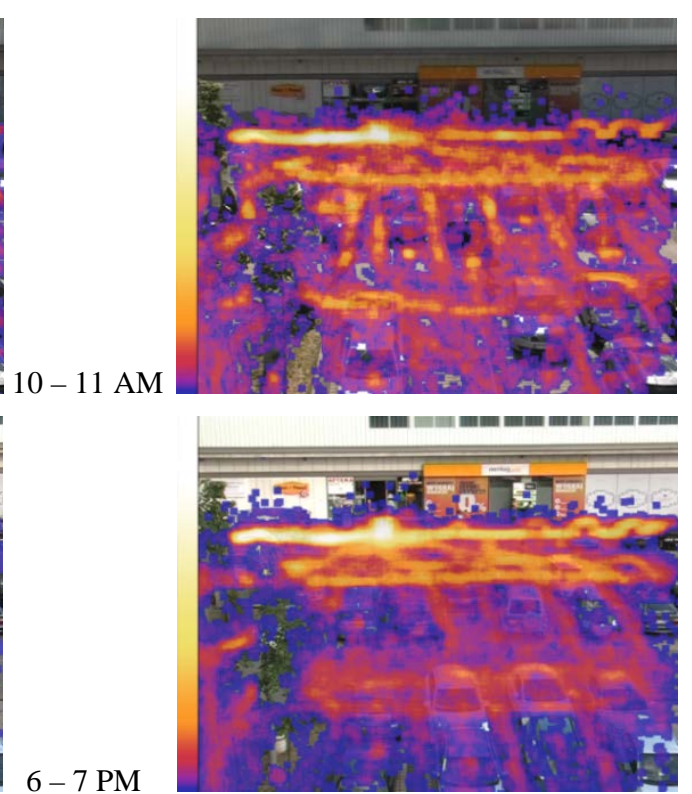

(b)

Figure 6. Selected examples of moving objects density maps: (a) TDM type and (b) CDM type both in one hour intervals taken between 6 AM and 7 PM 

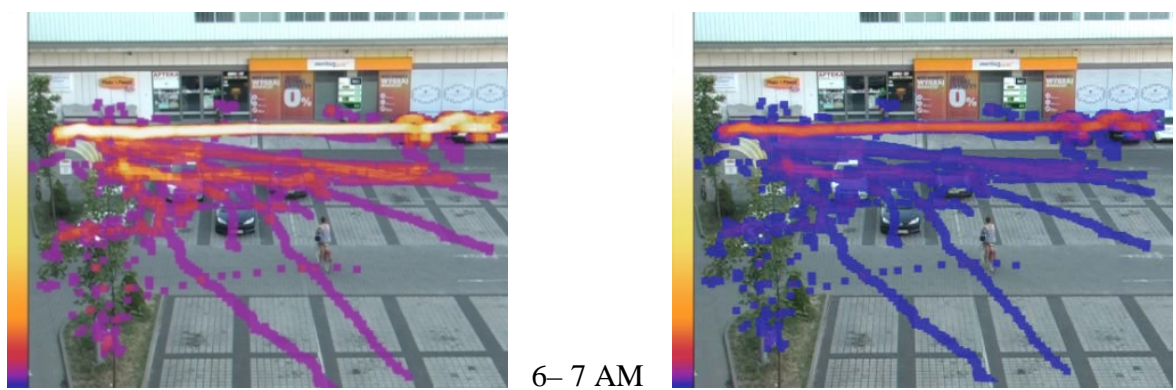

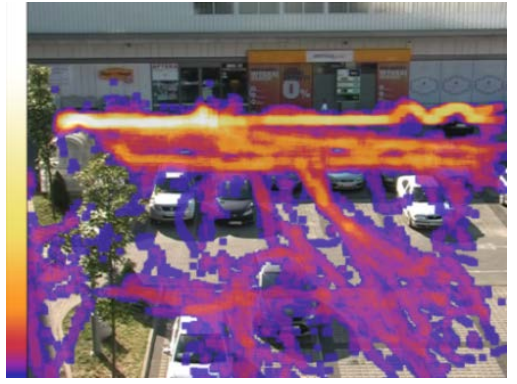

(a)
6- $7 \mathrm{AM}$

8- 9 AM

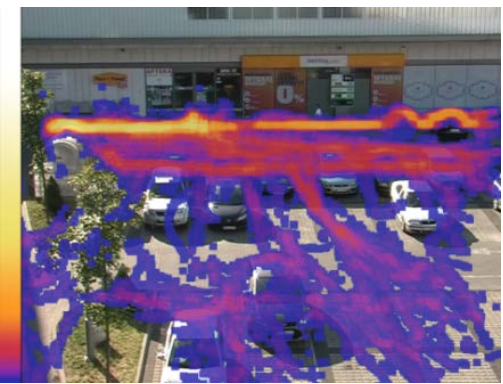

(b)

Figure 7. Example of density maps data normalization with the use of CDM: (a) before and (b) after normalization

\subsection{Determination of a "busy-hour"}

An analysis of density maps obtained on the basis of subsequent recordings from one location makes it possible to determine the busy or rush hour - the time the moving object density was the highest in the whole day. Fig. 8 shows the movement distribution changes in every hour and the busy-hour estimation based on the time and moving objects counts observation in a parking lot in front of the Green Point shopping center. The maximum length of time and the largest number of people in a given point was between 6 - 7 PM. During that hour in front of the entrance to the shopping center was 187 moving objects (people) and they were there for 195 s, i.e., $3.25 \mathrm{~min}$.

\section{Conclusions}

The presented two types of density maps of moving objects (people and vehicles) in prescribed areas of the observed scene: i.e. the presence of moving objects density maps and the counts of moving objects density maps occurred to be a very valuable tool for the analysis of the area exploitation, busy-hour estimation, detection of dangerous situations, and recognition of abnormal behavior. Normalization of the results (for example all over 
the day) is helpful for the estimation of the hour with the largest movement density (the rush or busy hour).

The proposed algorithms can be embedded in an intelligent CCTV system, which would offer automatic moving objects density analysis and reaction without any manual intervention or cumbersome analysis with questionnaires or with traditional traffic counting techniques.

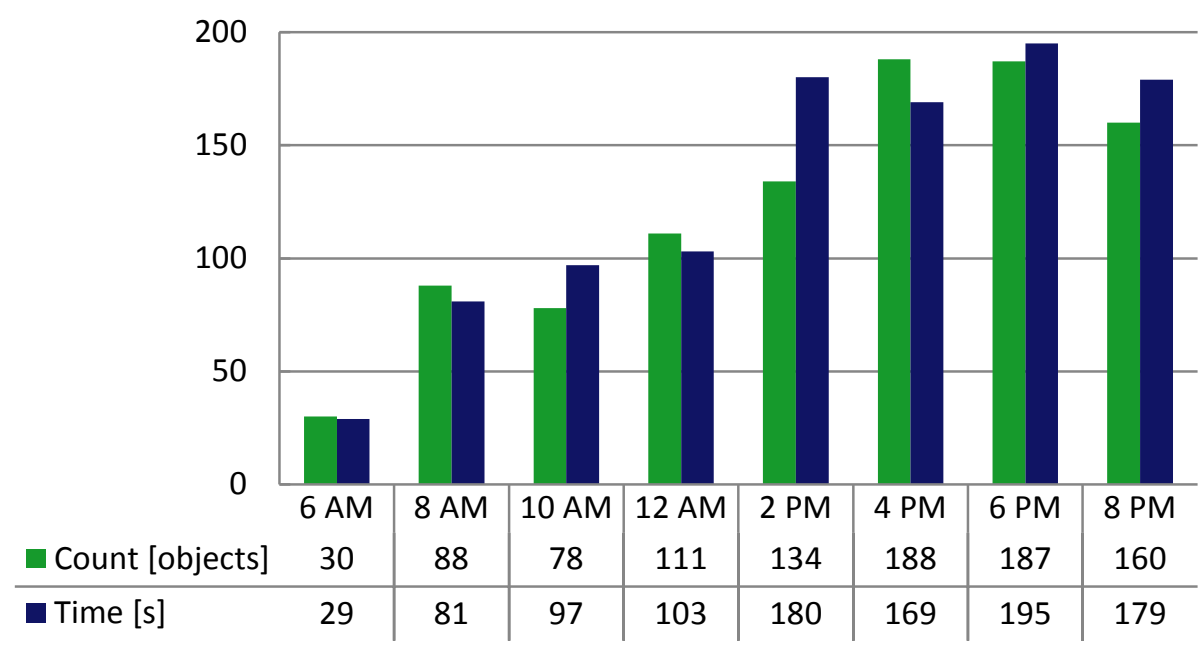

Figure 8. Busy hour estimation based on time and moving objects indexes (observation in the parking lot in front of the Green Point shopping center)

\section{Acknowledgment}

This work was partly supported by the DS project and partly by the Scholarship support for Ph.D. students specializing in the major strategic development for Wielkopolska, Sub-measure 8.2.2 Human Capital Operational Program, co-financed by the European Union under the European Social Fund.

\section{References}

[1] Bosch IVA 5.60 datasheet, http://resource.boschsecurity.us/documents/DS_IVA_5_60_Data_sheet_enUS_11858 668555.pdf, available at 2014-11-27

[2] Computer Vision Systems Toolbox for MatLab: http://www.mathworks.com/help/vision/index.html, available at 2014-05-30

[3] Computer Vision System Toolbox User’s Guide, MathWorks, 2015 vision.KalmanFilter class documentation (MatLab): 
http://www.mathworks.com/help/vision/ref/vision.kalmanfilter-class.html, available at 2015-03-20

[4] Computer Vision System Toolbox User's Guide, MathWorks, 2015

configureKalmanFilter function documentation (MatLab):

http://www.mathworks.com/help/vision/ref/configurekalmanfilter.html, available at 2015-03-20

[5] A. Czyżewski, P. Dalka, Moving Objects Detection and Tracking for the Purpose of Multimodal Surveillance System in Urban Areas, [in:] New Directions in Intelligent Interactive Multimedia Studies in Computational Intelligence, vol. 142, pp. 75-84, 2008. DOI: 10.1007/978-3-540-68127-4_8

[6] V. Einselein, H. Fradi, I. Keller, T. Sikora, J. Dugelay, "Enhancing Human Detection using Crowd Density Measures and an adaptive Correction Filter", IEEE International Conference on Advanced Video and Signal Based Surveillance, pp. 19-24, 2013.

[7] Heatmap tool from Cognimatics datasheet, http://www.cognimatics.com/downloads/trueview-heatmap-product-sheet.pdf, available at 2014-11-27

[8] K. Kopaczewski, M. Szczodak, A. Czyżewski, H. Krawczyk, "A method for counting people attending large public events", Multimedia Tools and Applications, 2013 (online). DOI: 10.1007/s11042-013-1628-0

[9] C. Lijun, H. Kaiqi, "Video-based crowd density estimation and prediction system for wide-area surveillance", China Communications, vol.10, no.5, pp.79-88, 2013

[10] S. F. Lin, J. Y. Chen, H. X. Chao, "Estimation of Number of People in Crowded Scenes Using Perspective Transformation", IEEE Transactions on Systems, Man, Cybernetics - Part A: Systems and Humans, vol. 31, no. 6, pp. 645-654, 2001.

[11] A. Marana, S. Velastin, L. Costa, R. Lotufo, "Estimation of crowd density using image processing", Image Processing for Security Applications, Digest No.: 1997/074, pp 11/1-11/8, 1997.

[12] T. Marciniak, A. Chmielewska, A. Dabrowski, A. Malina, "People counting vision system based on ARM processor programmed using Simulink environment", Electronics - constructions, technologies, applications, pp. 55-59, 2014.

[13] T. Marciniak, A. Dabrowski, A. Chmielewska, M. Nowakowski, "Real-Time Bi-Directional People Counting Using Video Blob Analysis" IEEE New Trends in Audio and Video / Signal Processing Algorithms, Architectures, Arrangements and Applications, pp. 161-166, 2012.

[14] MxAnalytix tool for map creation and people counting: http://www.mobotix.com/eng_AU/Support/Manuals/Software2/Compact-Guides, available at 2014-11-27.

[15] The NV-GS500 camera from Panasonic specifications:

http://panasonic.net/avc/camcorder/dv/global/products/nv-gs500/specifications.html, available at 2014-11-27

[16] M. Parzych, A. Chmielewska, T. Marciniak, A. Dąbrowski, "New Approach to People Density Estimation based on Video Surveillance", International SPA Conference, pp. 94-99, 2014.

[17] M. Parzych, A. Chmielewska, T. Marciniak, A. Dąbrowski, A. Chrostowska, M. Klincewicz, "Automatic people density maps generation with the use of movement detection analysis", International Conference on Human-System Interaction, pp.26-31, 2013. 
[18] M. Patzold, R. H. Evangelio, T. Sikora, "Counting people in crowded enviroments by fusion of shape and motion information", IEEE International Conference on Advanced Video and Signal Based Surveillance, pp. 157-164, 2010.

[19] PETS 2009 database: http://www.cvg.rdg.ac.uk/PETS2009/a.html, available at 2014-05-30

[20] H. Rahmalan, M. Nixon, J. Carter, "On Crowd Density Estimation for Surveillance", The Institution of Engineering and Technology Conference on Crime and Security, pp.540-545, 2006.

[21] M. Rodriguez, I. Laptev, J. Sivic, J. Audibert, "Density-aware person detection and tracking in crowds", IEEE International Conference on Computer Vision, pp. 24232430, 2001.

[22] H. Sharif, N. Ihaddedene, C. Djeraba, "Crowd Behavior Monitoring on the Escalation Exits:, International Conference on Computer and Information Technology, pp. 194200, 2008.

[23] Software for tracking shoppers: http://www.cnet.com/videos/software-tracksshoppers-to-find-stores-hot-zones/, available at 2014-11-27

[24] C. Stahlschmidt, A. Gavriilidis, A. Kummert, "Density Measurements from a TopView Position using a Time-of-Flight Camera", International Workshop on Multidimensional Systems, pp. 193-198, 2013.

Extended and revised version of work presented at the 18th IEEE SPA Signal Processing Conference, 22-24 September 2014, Poznan, Poland 